\title{
UNA COMPARACIÓN ENTRE LOS RESULTADOS ANTROPOLÓGICOS DE CINCO YACIMIENTOS DE ORIENTE ANTIGUO
}

\author{
Armando González Martín \\ Laboratorio de Poblaciones del Pasado \\ Departamento de Biología. Facultad de Ciencias \\ Universidad Autónoma de Madrid
}

\begin{abstract}
RESUMEN
El conjunto de resultados que nos ofrecen los estudios antropológicos sobre poblaciones de Oriente realizados en las últimas décadas proporcionan la posibilidad de abordar un análisis comparativo sobre los grupos humanos de la región. Este trabajo, que solo consiste en una primera aproximación, únicamente pretende reunir algunos resultados relevantes obtenidos en esas investigaciones.
\end{abstract}

Es indudable que queda mucho por conocer de estas poblaciones, pero hoy sabemos que el crecimiento en número de las investigaciones transdisciplinares que integran antropólogos de poblaciones del pasado en los grupos de trabajo es una realidad, hecho que proporciona un panorama esperanzador para el futuro próximo.

\section{PALABRAS CLAVE}

Antropología física, Arqueobiología, Arqueología funeraria, Paleodemografía, Paleopatología.

\begin{abstract}
The results that have been obtained in previous decades from anthropological studies of populations from the East provide the possibility for a comparative analysis on the human groups of the region. This study, which is only a first approximation, simply aims to collect relevant results obtained in these investigations.
\end{abstract}

Undoubtedly, there is much to know about these populations. Today, however, we know that an increase in the amount of multidisciplinary research, which integrates anthropologists specialized in past populations into work groups, is a reality. This fact provides a hopeful outlook for the near future.

\section{KEY WORDS}

Physical anthropology, Archaeobiology, Funerary archaeology, Paleodemography, Paleopathology.

\section{Introducción}

Aunque el vínculo entre Oriente y Antropología física es antiguo, un repaso a la literatura permite observar que si los estudios sobre poblaciones amplias son escasos, lo son aún más los estudios comparativos entre diferentes localidades o cronologías. Afortunadamente en las últimas décadas se han publicado distintos trabajos poblacionales de gran alcance, que permiten plantear hoy la posibilidad de realizar un análisis comparado entre algunas de esas poblaciones, con el objetivo de desvelar similitudes y diferencias entre los resultados antropológicos obtenidos por distintos autores.

Evidentemente no se trata de un metaanálisis exhaustivo, sino que se han elegido algunas colecciones bien estudiadas, con tamaños poblacionales importantes, sobre las que se han obtenido resultados y establecido conclusiones que abarcan, entre otros, los principales temas de trabajo sobre arqueobiología de las poblaciones del pasado: arqueología funeraria, paleodemografía, paleopatología, marcadores de actividad, paleodieta, etc., todos enfocados en el objetivo de conocer mejor su modo de vida.

Es sabido que disciplinas tan estrechamente relacionadas como Historia y Antropología de poblaciones del pasado, que durante largos periodos han mantenido una 
relación lejana, funden hoy a sus especialistas en equipos multidisciplinares quienes, con objetivos comunes, participan de forma conjunta en proyectos de investigación compartidos. Eso permite a los antropólogos participar en muchas ocasiones también sobre el terreno, aprovechando la oportunidad de estudiar los restos óseos humanos en su contexto cultural, como proponen los principios de la llamada Antropología de campo ${ }^{1}$.

No obstante, hay quien opina que el conocimiento de la biología de aquellos grupos humanos es todavía marginal, realizándose estudios muy generales (determinaciones del sexo y estimaciones de talla y de edad de muerte), a veces sobre esqueletos individuales o sobre muestras insuficientes, incluyendo solo excepcionalmente estudios demográficos o patológico/epidemiológicos ${ }^{2}$. Parece pertinente en esta situación plantear el presente trabajo, teniendo en cuenta que, por su limitado alcance, será solamente un punto de partida para futuras comparaciones, que puedan abarcar tanto otros grupos humanos no incluidos aquí como todos aquellos que vayan estudiándose a partir de ahora.

\section{Ur (Irak)}

Originalmente excavados por Charles Leonard Woolley en los años '20 del siglo pasado, los cementerios de Ur han proporcionado cientos de tumbas, aunque la atención parece haberse centrado especialmente en las llamadas tumbas reales, de monumentales proporciones y ricos ajuares, en algunos casos con decenas de individuos. Los estudios antropológicos parecen tener un peso muy limitado, ya que solo se han encontrado estudios relativos a no más de algunas decenas de individuos de distintos periodos. El primero de esos estudios, en un enfoque muy propio de la época se centra es aspectos tipológicoraciales, salpicados con afirmaciones que sería difícil encontrar, por cuestiones obvias, en ningún estudio actual:

Of particular interest is it to observe the fine physique and the rich brain endowment of Queen Shub-ad and of the Prince, Mes-kalam-dug. The latter was an exceptionally strong man physically, and if we may rely on size of brain as an index of mental capacity-then was the Prince not only physically strong but also a man of superior capacity. The Queen's cerebral endowment was exceptional, and if we can trust physical development of the body as a clue to sexual mentality, then we may infer that she was also a very feminine woman ${ }^{3}$.

Más recientemente se realiza el estudio ${ }^{4}$ que proporciona más información poblacional, si bien su alcance es escaso en comparación con el número de restos humanos encontrados. Desde el punto de vista del grupo humano este trabajo aporta algunas conclusiones que deben ser tenidas en cuenta. No se realiza por el exiguo tamaño muestral $(\mathrm{N}=35)$ un estudio paleodemográfico, pero se describen esqueletos de todas las clases de edad, desde fetales hasta seniles, estando representados tanto hombres como mujeres. Su datación se determina entre 4000 y 600 a. C. y se indica como posición de enterramiento predominante la de decúbito lateral flexionado. También se da cuenta de un notable dimorfismo sexual, con una talla para las mujeres entre 1,51 y 1,60 m y entre 1,64 y 1,72 m para los hombres, es decir, sin superposición en sus distribuciones. Las evidencias relacionadas con las actividades destacan un desarrollo muscular notable fruto de una actividad física intensa, observada desde la infancia y que en algún momento se relaciona con la esclavitud. Mucho menos evidentes son estos marcadores

\footnotetext{
Duday, 1997

Soltysiak, 2006

3 Keith, 1934

4 Molleson y Hodgson, 2003
} 
de actividad en las tumbas reales, interpretándose este hecho como consecuencia de una clara estratificación de la sociedad. Al menos en algunos periodos hay signos de alimentación deficiente, proponiéndose diagnósticos de enfermedades carenciales como anemia o raquitismo. La patología es escasa, especialmente la dental, especificándose que son ocasionales las caries, los abscesos, la enfermedad periodontal y la pérdida dental premortem.

Trabajos más recientes se han centrado en el aspecto concreto de los sacrificios humanos, de los que se encuentran varias evidencias tanto en Ur como en otros yacimientos de la región ${ }^{5}$. Otros aspectos destacables de los aparentemente complejos rituales mortuorios tienen que ver con el proceso de calentado, quemado o ahumado de los cadáveres con la intención de evitar o ralentizar la putrefacción, y la utilización de sales de mercurio (cinabrio) en lo que constituiría su uso más antiguo fuera del oriente lejano en el proceso de tratamiento funerario 6 .

En línea podemos encontrar la página web $U r$ Online $^{7}$, producida conjuntamente por The British Museum y Penn Museum con el apoyo de diferentes patrocinadores, en cuyo catálogo podemos encontrar 22 objetos bajo la denominación "human bones". Todo ello da una idea de cuánto desconocemos todavía de este grupo humano desde el punto de vista antropológico, a pesar del tiempo transcurrido desde su descubrimiento.

\section{Abu Hureyra (Siria)}

Los restos recuperados por Andrew M. T. Moore en los años '70 del siglo pasado fueron estudiados por Molleson ${ }^{8} \mathrm{y}$ dieron lugar a un conocimiento bastante profundo del modo de vida alrededor de la transición neolítica (9500-6500 a. C.). Entre los 162 individuos estudiados se cuentan tanto mujeres como hombres y no-adultos, estos últimos en una proporción con los adultos cercana a 1:1, lo que sugiere la idea de una fecundidad y una mortalidad infantil elevadas. Enterraban a sus muertos en tumbas excavadas fuera de los lugares de habitación, pero también en las propias casas, donde son más numerosos los enterramientos femeninos.

Se describe la buena salud general de la población, pero con una acumulación de signos degenerativos, ya desde la juventud, que unidos a los marcadores de fuerte musculatura podrían estar relacionados con intensas tareas cotidianas, entre las que probablemente estuviera la carga de pesos. La columna vertebral sufriría especialmente con estas actividades, tanto las cervicales con el transporte de cargas sobre la cabeza como las dorso-lumbares con la molienda manual de cereales. Las articulaciones acababan desarrollando signos degenerativos de tipo artrósico a base de soportar las tensiones que suponían estas actividades físicas intensas.

Los signos patológicos que se asocian a la molienda de cereales parecen concentrarse en los individuos femeninos, algo que supone la existencia de una división de papeles en esa sociedad. Las molederas dejarían un residuo en la propia harina que contribuyó a un desgaste dentario generalizado y claramente observable. Actividades como la cestería y la fabricación de esterillas, en las que el auxilio de la boca pudo ser habitual, contribuyeron también a incrementar ese desgaste, aunque en este caso se trataría de surcos solo observables en determinadas piezas dentales. El desarrollo de la fabricación alfarera terminó con alguna de estas actividades, lo que se refleja en la desaparición de algunos de los signos anteriormente descritos en las últimas fases del cementerio.

\footnotetext{
Recht, 2010; Vidale, 2011

6 Baadsgaard et al., 2011

7 http://www.ur-online.org/

8 Molleson, 1994
} 
No es esta la única característica de los periodos cerámicos, en los que la proporción de bebés aumenta con respecto a los niveles anteriores, algo que puede interpretarse como un aumento de la natalidad o como una intensificación de la mortalidad infantil. Se propone que el aumento de la población pudo facilitar también la transmisión de patógenos y favorecer la aparición de signos de infecciones. El cocinado de los cereales pudo estar detrás del aumento de la frecuencia de caries en esta fase.

\section{Çatalhöyük (Turquía)}

Excavada inicialmente en los años '60 del pasado siglo por James Mellaart, sigue siendo objeto de nuevas campañas e investigaciones ${ }^{9} \mathrm{y}$ ha dado lugar a una literatura muy extensa, centrada también en aspectos funerarios y antropológicos. Sus enterramientos, hasta hoy más de 600, tienen como característica general encontrarse bajo el suelo de las propias casas, siguiendo patrones de distribución determinados en función de la edad de los sujetos (adultos preferentemente al $\mathrm{N}$, bebés hacia el $\mathrm{S}$ ) y en ocasiones alterados de forma aparentemente intencional.

Se han realizado varias investigaciones relativas a diferentes conjuntos de restos humanos, tanto pertenecientes a las primeras campañas de excavación ${ }^{10}$ como centradas en los 94 individuos recuperados de 49 tumbas entre 1995 y $1999^{11}$, aunque el trabajo más extenso y reciente, que se usará aquí como referencia principal, incluye 382 individuos relativamente completos ${ }^{12}$.

En el trabajo citado se describe una población humana en rápido crecimiento demográfico, que descansa en un aumento de la fertilidad y de los nacimientos, producto en último término de la domesticación, la intensificación agrícola y el consecuente sedentarismo. A través del estudio de los restos óseos se concluye que el cambio de estilo de vida acaba suponiendo un incremento de la morbilidad y un aumento de la carga de trabajo. El crecimiento y desarrollo de los individuos juveniles y la talla media adulta reflejan ajustes a las condiciones locales.

Las estimaciones sobre el tamaño máximo de la población de origen oscilan entre 3500 y 8000 miembros. El patrón general de los enterramientos es el de depósitos individuales y primarios en fosas ovaladas excavadas en el suelo de las casas, con esqueletos en posición flexionada, algunos de los cuales probablemente fueran previamente empaquetados $\mathrm{y}$, solo unos pocos, acompañados por diversos elementos de ajuar (conchas, madera, ornamentos, fauna, etc.). Algo más del $50 \%$ de los individuos son no-adultos ( 0 a 3 años: 34 \%; 3 a 19 años: 23 \%) (Figura 1). Este elevado índice de juventud de la población habría sido interpretado hasta tiempos recientes como resultado de una intensa mortalidad infantil, aunque hoy sabemos que la frecuencia de no-adultos en los cementerios está más relacionada con la natalidad que con la mortalidad. La proporción de sexos es equilibrada, aunque más de un $20 \%$ de los adultos quedan sin sexar.

\footnotetext{
9 Véase http://www.catalhoyuk.com/

10 Se citan en varias fuentes como los trabajos pioneros realizados sobre los restos humanos excavados por Mellart los de Angel (1971) y Ferembach (1972 y 1982).

11 Andrews y Bello, 2006; Andrews et al, 2005

12 Larsen et al., 2015
} 


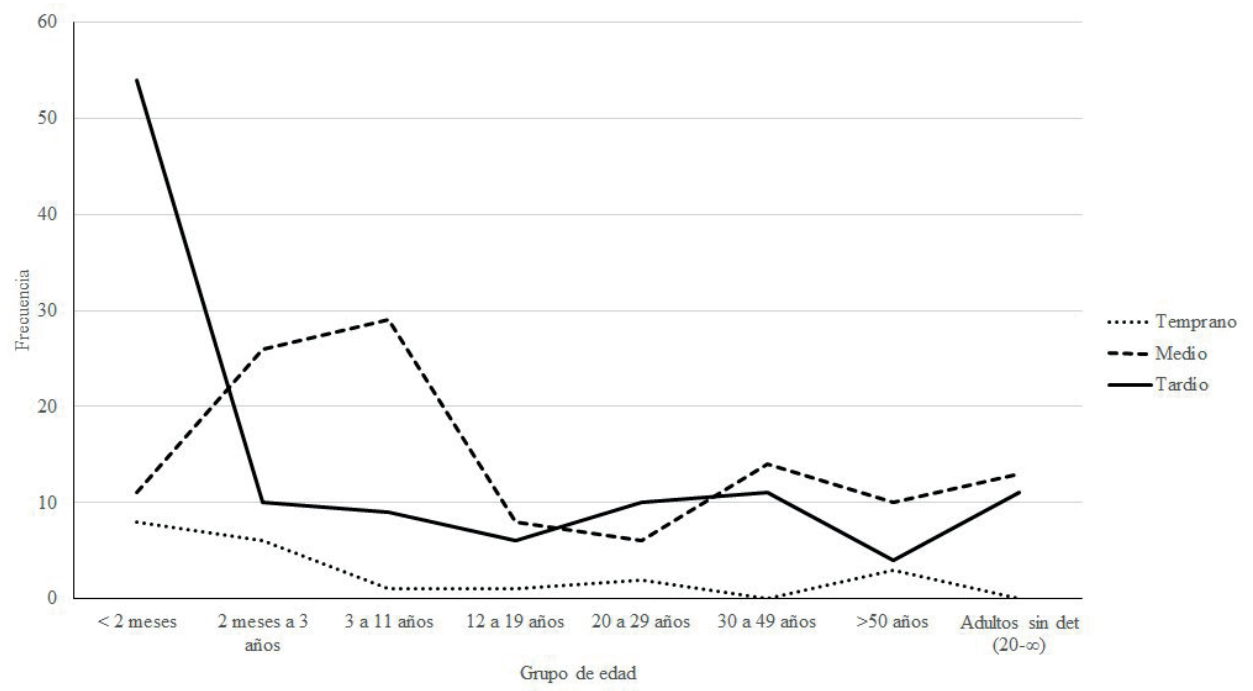

Figura 1 - Frecuencia de individuos por grupo de edad en Çatalhöyük por periodos (Datos de Larsen et al., 2015).

La disponibilidad de trigo, cebada y cabra, inferida de los restos biológicos no humanos, se traduce a su consumo a través del estudio de los isótopos estables de carbono y nitrógeno. En ninguno de los periodos específicos se encuentran diferencias significativas en la dieta entre sexos, determinándose que el proceso de destete de los bebés comenzaría hacia el año y medio, culminándose hacia los tres. La frecuencia de caries, relacionada directamente con el consumo de hidratos de carbono cariogénicos, ofrece resultados también consistentes con la ausencia de diferencias dietéticas entre sexos.

La media de estatura se estima entre 1,50 y $1,55 \mathrm{~m}$ para las mujeres y rondaría 1,65 m para los hombres, valores normales en relación a las poblaciones utilizadas como comparación. La estimación del peso corporal (entre 50 y $60 \mathrm{~kg}$ para las mujeres y de 60 a $70 \mathrm{~kg}$ para los hombres) también ofrece valores similares a los de otras poblaciones. El crecimiento y desarrollo de los no-adultos parece ser normal. En cuanto al estilo de vida, los autores analizan indicadores patológicos relacionados con la carga de trabajo, como los signos artrósicos a nivel axial y articular, concluyendo en la existencia de un aumento con la edad de muerte de los individuos, la ausencia de diferencias sexuales y una disminución de la frecuencia en el último periodo de uso del yacimiento.

Tal vez uno de los resultados más sorprendentes sea la baja afinidad biológica encontrada, analizada a través de caracteres fenotípicos dentales, entre los individuos enterrados en las mismas casas, contradiciendo la hipótesis inicial relativa a las relaciones familiares cercanas entre ellos.

\section{Jebel al-Buhais (EAU)}

De todos los restos recuperados en el Emirato de Sharjah, el conjunto procedente del cementerio neolítico Buhais 18 (5100-4300 a. C.) destaca por el volumen de la colección (más de 450 individuos) y por la profundidad del estudio realizado sobre ellos ${ }^{13}$. Se trata de enterramientos tanto primarios -semiflexionados, preferentemente en decúbito lateral derecho y orientados hacia el norte- como secundarios, en ocasiones formados solo por cráneos que miran hacia el este y conjuntos de huesos largos. Los enterramientos primarios se encuentran asociados frecuentemente a ornamentos personales, en muchas ocasiones fabricados en concha. Algunos restos fueron quemados, observándose coloración negra, típica de temperaturas poco elevadas $\left(300-400^{\circ} \mathrm{C}\right)$.

13 Uerpmann et al., 2006 
El estudio paleodemográfico muestra una población en la que solo dos de cada tres individuos alcanzaba la edad adulta, con una esperanza de vida al nacimiento de 27,5 años, algo mayor para los hombres que para las mujeres. Su perfil de probabilidad de muerte representa algo similar a lo que cabe esperar de un grupo humano sometido a una mortalidad natural, aunque los restos de los infantiles más pequeños ( 0 a 4 años: 10,8 $\%)$ son demasiado escasos. La proporción de sexos es equilibrada, aunque no es posible determinar esta variable para todos los individuos recuperados por las condiciones de su preservación.

Las características morfológicas de los restos muestran un grupo en general poco robusto. El dimorfismo sexual es moderado. La talla media de las mujeres se estima en 1,60 m, siendo la de los hombres de 1,71 m. Los traumatismos craneales son frecuentes (14\%), siendo doble su proporción en masculinos que en femeninos. Su localización es preferentemente frontal o parietal y se interpretan como evidencias de la existencia de enfrentamientos interpersonales. Es posible que los tres casos de trepanaciones encontrados puedan estar relacionados también con traumatismos. Sin embargo, los traumas poscraneales son escasos (doce casos) y se concentran en los miembros superiores.

Aunque la deficiente preservación siempre limita las posibilidades de identificación de caracteres patológicos, se describen signos artrósicos tanto a nivel articular como axial, siendo mucho más frecuente su existencia en la columna cervical. Hay alteraciones que demuestran la existencia de enfermedades infecciosas, particularmente frecuentes entre los infantiles, y se diagnostican enfermedades metabólicas como la gota (cuatro casos). Los indicadores de deficiencias nutricionales son escasos, igual que los signos patológicos bucodentales -no se han observado caries-. También se encuentran enfermedades de tipo tumoral como los osteomas craneales (siete casos) y anomalías congénitas como la escafocefalia, consistente en el cierre prematuro de la sutura craneal sagital (dos casos). Todos estos datos sirven a los autores para proponer unas buenas condiciones de vida, en general mejores que las del resto de poblaciones utilizadas como comparación.

En tiempos recientes se ha publicado en red un catálogo interactivo relativo al cementerio ${ }^{14}$, que incluye la información antes publicada y nuevos datos (arqueológicos, osteológicos e isotópicos) para la reconstrucción de la vida de este grupo humano. Todos los datos e imágenes están a disposición de cualquier persona interesada en el sitio web citado, así como una relación exhaustiva de las referencias bibliográficas existentes.

\section{Tell Halula (Siria)}

Se trata de un yacimiento cuyo estudio está a cargo de la Misión Arqueológica Española desde los años 90, encabezada por un equipo de la Universidad Autónoma de Barcelona $^{15}$. Situado en el valle del Éufrates, con una datación aproximada entre 6700 y 4500 a. C., el área excavada hasta la fecha parece ser una parte muy pequeña de la superficie total del yacimiento.

La memoria citada, con una extensión cercana a las 1000 páginas, dedica un centenar de ellas a la arqueología funeraria y los restos humanos. La totalidad de las sepulturas documentadas hasta la fecha, caracterizadas por su gran regularidad y homogeneidad, están en el interior de las unidades domésticas, con tendencia a concentrarse en la entrada de la habitación principal de cada casa en forma de fosas circulares, de paredes rectas y base plana o cóncava, conteniendo inhumaciones individuales y primarias. Los depósitos parecen haber sido realizados en espacio vacío, presentando mayoritariamente cubiertas de tapial o adobe. Aproximadamente la mitad conservan restos de elementos protectores (saco, tejido, esteras o cestería, a veces combinados). Se han aislado un total de 182 individuos,

\footnotetext{
${ }^{14} \mathrm{https} / /$ publikationen.uni-tuebingen.de/xmlui/static/html/EXTERN/Catalog_BHS18/gallery/index.html\# ${ }^{15}$ Molist, 2013
} 
de los que la mayor parte fueron depositados en posición sedente y orientados hacia el sur o hacia el oeste. Más de la mitad contienen elementos de ajuar, que aparecen en mayor número acompañando a los individuos infantiles. Entre ellos se citan sílex, obsidiana, ocre, hueso, bolas de cal con improntas de tejido y ornamentos -collares, brazaletes, diademas y cinturones-.

El estudio antropológico da idea de un estado de preservación no óptimo, ya que solo ha sido posible estimar la edad de muerte del $80 \%$ y obtener valores antropométricos de una treintena. Tres de cada cinco individuos no alcanzaron la edad adulta, lo que se atribuye a una elevada mortalidad infantil y juvenil. La estatura estimada, que no se diferencia por sexos ${ }^{16}$, se sitúa entre 1,62 y 1,69 m. A través de indicadores patológicos como la cribra orbitalia, con una prevalencia superior en los infantiles y juveniles, se infieren unas condiciones de vida duras y difíciles, salpicadas de malnutrición e infección. Se cita también la existencia de hiperostosis porótica.

Tanto mujeres como hombres son calificados como robustos, con zonas de inserción muscular marcadas tanto en los miembros superiores como en los inferiores. Sin embargo, la artrosis, muchas veces interpretada como resultado del deterioro esquelético relacionado con la actividad física, parece ser poco frecuente. La prevalencia de traumatismos también es baja. No existen otros signos patológicos degenerativos ni tumorales. Se citan evidencias de la práctica de trepanaciones que, de confirmarse, podría tratarse de algunas de las más antiguas documentadas. Las caries y la pérdida dental premortem son poco frecuentes, no así la hipoplasia del esmalte dental, un defecto en su formación que se observa en cinco de cada seis individuos.

Estudios moleculares han sido aplicados para la determinación del sexo, ayudando a dicha determinación cuando el estudio morfológico no era concluyente, y para la caracterización genética de la población. Salvo en casos puntuales, los estudios de la línea paterna (cromosoma Y) y materna (ADN mitocondrial) indican que no hay relación familiar cercana entre los individuos enterrados en cada casa. Lamentablemente la dificultad técnica de este tipo de análisis hace que su alcance haya sido escaso en comparación con el número total de individuos estudiados. Algunas conclusiones van en la línea de su baja relación con la actual población europea y la existencia de linajes de origen africano, probablemente relacionados con flujos de población mucho más antiguos.

\section{Reflexiones finales}

Como se citó en la introducción, nuestro conocimiento de las poblaciones de Oriente parece ser todavía marginal. Hay que tener en cuenta que todos los resultados aquí presentados han sido obtenidos de un total de poco más de un millar de esqueletos estudiados, con lo que difícilmente podríamos considerar que la muestra en conjunto pueda ser representativa de un periodo tan dilatado y una extensión tan grande.

No obstante, se han querido reunir aquí algunos resultados y conclusiones de los estudios más importantes realizados hasta la fecha por su interés para conocer más de la biología y la tradición funeraria de estos grupos humanos, no con la intención de llevar a cabo un estudio comparativo exhaustivo, sino de proponer un punto de partida para estudios de mucho más alcance. Es previsible que en un futuro inmediato este tipo de estudios siga aumentando en número, y que la participación de antropólogos en las investigaciones sirva para obtener nuevos datos. Las posibilidades que ofrecen herramientas como internet para la divulgación de resultados pueden contribuir también a aumentar las posibilidades de realizar estudios de tipo comparativo.

16 Otro trabajo centrado en estos restos (Estebaranz et al., 2007) describe un dimorfismo sexual casi nulo para la talla 


\section{Referencias bibliográficas}

Andrews, P., Molleson, T., Boz, B., 2005, "The Human Burials at Çatalhöyük", in Hodder I (ed.), Inhabiting Çatalhöyük: Reports from the 1995-1999 Seasons, Çatalhöyük Project Vol. 4, Cambridge, pp. 499-520.

Andrews, P., Bello, S., 2006, "Pattern in human burial practice", in Gowland, R. Knüsel, C. (eds.), Social archaeology and funerary remains, Oxford, pp. 14-29.

Baadsgaard A., Monge J., Cox S., Zettler R., 2011, "Human sacrifice and intentional corpse preservation in the Royal Cemetery of Ur" Antiquity 85(327), pp.27-42.

Duday H., 1997, “Antropología biológica "de campo", tafonomía y arqueología de la muerte", en Malvido E., Pereira G., Tiesler V (eds.) El cuerpo humano y su tratamiento mortuorio, México DF, pp. 91-125.

Estebaranz, F., Martínez, LM., Anfruns, J., Martínez, A., 2007, “Estudio preliminar del esqueleto postcraneal del yacimiento neolítico de Tell Halula, Siria", en Justel, JJ., Solans, BE., Vita, JP., Zamora, JA. (eds.) Las aguas primigenias. El Próximo Oriente Antiguo como fuente de civilización. Zaragoza, pp. 401-420.

Keith A., 1934, "Report on human remains", in Woolley CL., Ur excavations. Vol. II: The Royal Cemetery. NY, pp. 400-409.

Larsen CS., Hillson SW., Boz B., Pilloud MA., Sadvari JW., Agarwal SC., Glencross B., Beauchesne P., Pearson J., Ruff CB., Garofalo EM., Hager LD., Haddow SD., Knüsel CJ., 2015, "Bioarchaeology of Neolithic Çatalhöyük: Lives and Lifestyles of an Early Farming Society in Transition", Journal of World Prehistory 28(1), pp. 27-68.

Molist M., (coord.), 2013, Tell Halula: un poblado de los primeros agricultores en el valle del Éufrates, Siria. Memoria Cientifica, Madrid.

Molleson T., 1994, "La lección de los huesos de Abu Hureyra", Investigación y Ciencia 217, pp. 60-65.

Molleson,T., Hodgson, D, 2003, “The human remains from Woolley's excavations at Ur" Iraq 65, pp. 91-129.

Recht, L., 2010, "Human sacrifice in the ancient Near East", Trinity College Dublin Journal of Postgraduate Research 9, pp. 168-180.

Soltysiak A., 2006, "Physical anthropology and the "Sumerian problem", Studies in Historical Anthropology 4, pp. 145-158.

Uerpmann HP., Uerpmann M., Jasim SA., 2006, Funeral monuments and human remains from Jebel al-Buhais. Sharjah.

Vidale M., 2011, "PG 1237, Royal Cemetery of Ur: Patterns in Death", Cambridge Archaeological Journal 21(3), pp. 427-451. 